\title{
The effect of contact interface on dynamic characteristics of composite structures
}

\author{
James Shih-Shyn $\mathrm{Wu}^{\mathrm{a}, *}$, Jyh-Cheng Chang ${ }^{\mathrm{a}}$, Jui-Pin Hung ${ }^{\mathrm{b}}$ \\ ${ }^{a}$ Institute of Mechanical Engineering, National Chung-Hsing University, Taichung, Taiwan 40227, ROC \\ ${ }^{\mathrm{b}}$ Department of Mechanical Engineering, National Chin-Yi Institute of Technology, Tai-Ping, Taichung, Taiwan 320, ROC \\ Received 20 May 2005; received in revised form 23 May 2006; accepted 4 July 2006 \\ Available online 25 September 2006
}

\begin{abstract}
In this project, nonlinear characteristics on the rolling interface of a linear guide were studied by the finite element analysis and experimental verification. Contact of the ball/surface rolling interface in the rolling guides was simulated as a three-dimensional membrane element without thickness. By introducing Hertzian contact theory and applying proper normal/shear stiffness to such contact elements in the overall finite element model, dynamic behaviors of linear guides affected by preload were thus investigated. In the finite element procedure, three contact models, 1D point-to-point, 2D point-to-point and 3D surface-to-surface, were sequentially introduced for purpose of verification with experiments. As a validation in this project, vibrational experiments on linear guides with different preloads were conducted and related frequency spectrums were derived. Both the finite element and the experimental results reveal that the natural frequency of a linear guide increases with the increment of the preload. In addition, the dynamic characteristics predicted by finite element analysis agree well with those measured from instrumental experiments. The proposal of current study may provide an alternate and reliable way for understanding of the dynamic characteristic of the rolling contact components in machine design field.
\end{abstract}

(C) 2006 IMACS. Published by Elsevier B.V. All rights reserved.

Keywords: Linear guide; Hertz theory; Contact element

\section{Introduction}

Following the development in scientific technology, the requirement of the high-precision machine has been increasing steadily. In order to achieve the high-speed and high-precision positioning, the static/dynamic behaviors of transmission systems must be sufficiently understood in the design stage for a precision machine. The linear guide system with rolling balls is the more popular and effective transmission system because of the low wear and friction force induced at ball grooves when compared to the conventional guide with sliding contact interface. For the analysis of the static/dynamic behaviors, a linear spring was usually used to simulate the contact characteristics of the ball between the carriage and guideway (Ohta [10]). It is well known from Hertz's contact theory that the elastic deformation between the carriage and rolling ball or between balls and the rail behaves a nonlinear way with the contact force. Therefore, the nonlinear simulation of the contact interface of a linear guide system is worthy for investigation.

\footnotetext{
* Corresponding author. Tel.: +886 $422870195 x 304$; fax: +886 422877170.

E-mail address: sswu@dragon.nchu.edu.tw (J.S.-S. Wu).
} 
Applications of the Hertz's contact theory have been published in many earlier studies. Hagiu and Gafitanu [3] applied Hertz's theory to derive the relation between the normal force and the deformation of the ball bearing, and obtained the dynamic characteristic of the machine tools. Perret-Liaudet $[11,12]$ described a shooting method to solve for the resonance of the sphere-plan contact. Yeh and Liou [14] showed the concept and estimation of virtual spring elements. Moreover, Lynagh et al. [7] and Hernot et al. [4] employed the nonlinear Hertz's contact theory to discuss the vibration and the stiffness matrix of the ball bearings. Recently, Monsak Pimsarn and Kazem Kazerounian [13] presented a PISE (pseudo-interference stiffness estimation) method to evaluate the equivalent mesh stiffness quickly. Concerning the studies relating to linear guide system, Ohta [10] employed the energy balance and Lagrange's method to derive the natural frequency of the carriage block in a linear rolling guide, and proved that the main peaks of the spectra were caused by lower rolling, yawing, pitching, vertical and higher rolling of the natural vibration modes. Later on, Ohta and Hayashi [9] proved that the linear guide system could be progressively analyzed by the finite element method. Different vibration modes including the lower rolling, yawing, pitching, vertical, first flexural, second flexural, and third flexural natural frequency were found in such a rolling contact mechanism. Meanwhile, it was noted that in Ohta and Hayashi's work, the contact stiffness was simulated by a single spring element only in normal contact direction, which might be referred to an one-dimensional point-to-point contact mode. So far, no study related to the surface-to-surface contact nonlinear model has been reported. This is a notable shortcoming because the surface-to-surface contact model is considered to be able to simulate the real contact behaviors.

In the study, finite element models to simulate the contact characteristic of the rolling interfaces were developed and discuss how the dynamic characteristic of the linear guide system was affected by the stiffness of the contact interface and the equivalent stiffness and the nonlinear responses were evaluated based on Hertzian elastic contact theory. In the beginning of this report, through Lagrange's approach, the difference of frequencies were discussed between the 1D point contact model presented in relevant literatures and 2D point-to-point contact model proposed in this study (Egert [1]). Moreover, Hertzian interface idea was adopted in the contact model between the rolling ball and carriage/rail for deriving the stiffness of the contact element to understand the dynamic behaviors of the linear guide system. The rolling track was also simulated in a realistic way by incorporating the finite element analysis with the surface-surface contact model. As a validation, relevant experimental modal tests were carried out on linear guideways with different preloads. Finally, comparisons and discussions on the results obtained from numerical predictions and experimental measurements were made and suggestions for further studies on the factors affecting the vibrations characteristics of rolling guides were supplied at the end of this report.

\section{Contact stiffness of rolling interface}

The linear guideway system is essentially designed with a Gothic arc groove, as shown in Fig. 1, which enables the rolling ball to contact with carriage and rail simultaneously and can be considered as a Hertzian contact mode here. According to the Hertzian elastic contact deformation theory, there is a nonlinear relationship between the local deformation at the contact point and the applied load acted on the contact components. For a linear guideway, the deformation of the raceway groove will increase with the applied load on the ball and enable the contact stiffness of the rolling interface to rise in a nonlinear way. Such a variation in contact stiffness may affect the dynamic behavior of this mechanism to a different extent. Therefore, in order to obtain correct vibration characteristics of a guideway, the contact stiffness must be suitably defined, see Johnson [6] and Goldsmith [2]

Fig. 1 shows the geometry of the ball in contact with the groove of carriage and rail at the contact angle of $\beta$, forming a two-point contact state. When a compression force $\mathrm{F}$ is applied, the contact boundary of contacting objects will deform a small amount of $\alpha$ and form an area contact of the shape of an ellipse with the major axis $2 a$ and minor axis $2 b$. The relationship between local contact force $F$ and elastic deformation $\alpha$ can be written as follows:

$$
\begin{aligned}
F & =k_{h} \alpha^{3 / 2} \\
k_{h} & =\frac{4}{3} \frac{q_{k}}{\left(\delta_{1}+\delta_{2}\right) \sqrt{A+B}} \\
\delta_{i} & =\frac{1-\mu_{i}^{2}}{\pi E_{i}}
\end{aligned}
$$




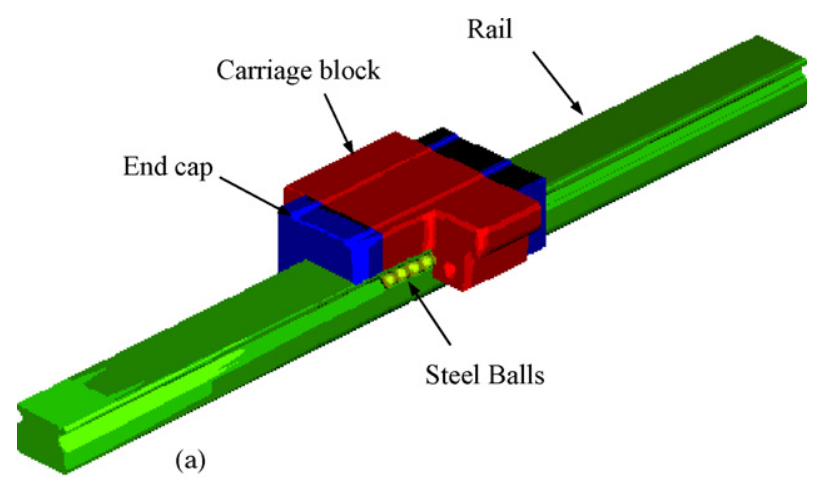

(a)

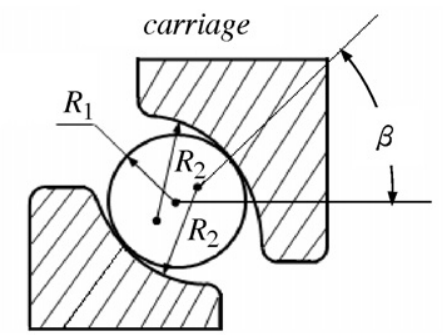

(b)

Rail

Fig. 1. (a) Schematic of a linear guide system and (b) geometry of the rolling ball in contact with rail and carriage.

$$
\begin{aligned}
& a=q_{a} \sqrt[3]{\frac{3 F\left(\delta_{1}+\delta_{2}\right)}{4(A+B)}} \\
& b=q_{b} \sqrt[3]{\frac{3 F\left(\delta_{1}+\delta_{2}\right)}{4(A+B)}}
\end{aligned}
$$

where $\alpha$ is the elastic deformation of the contact area, $\delta_{i}$ the material properties of Hertz's contact theory, $E$ the Young's modulus, $\mu$ the Poisson's ratio of material, $a$ the semi-major and $b$ is the semi-minor of the contact ellipse. Constants $A$, $B, q_{a}, q_{b}$, and $q_{k}$ were determined according to Goldsmith [2] where the configuration of contact of a linear guideway can be simplified as a sphere with radius $R_{1}$ to a cylindrical cup with radius $R_{2}$, that is,

$$
A=\frac{1}{2}\left(\frac{1}{R_{1}}-\frac{1}{R_{2}}\right), \quad B=\frac{1}{2 R_{1}},
$$

From Eq. (1), the normal stiffness can then be obtained as:

$$
K_{n}=\frac{\mathrm{d} F}{\mathrm{~d} \alpha}=\frac{3}{2} k_{h} \alpha^{(1 / 2)}
$$

As revealed in Hwang and Gahr [5], the stiffness in either the normal or the tangential direction is governed by material properties of the bodies in contact, normal load, lubricant and surface roughness. In this study, the tangential stiffness $K_{\mathrm{S}}$ associated with frictional effect is therefore correlated with the normal stiffness $K_{\mathrm{n}}$ following the Columb's friction law.

\section{Modeling of the linear guideway system}

\subsection{Analytical approach}

In mathematical modeling for the contact structure, the linear guideway is considered as a spring-mass system in which the rail and carriage are taken as rigid bodies and connect a series of spring elements with adequate axial stiffness. The coordinate system and vibration modes of the carriage are illustrated in Fig. 2a, where the origin of 

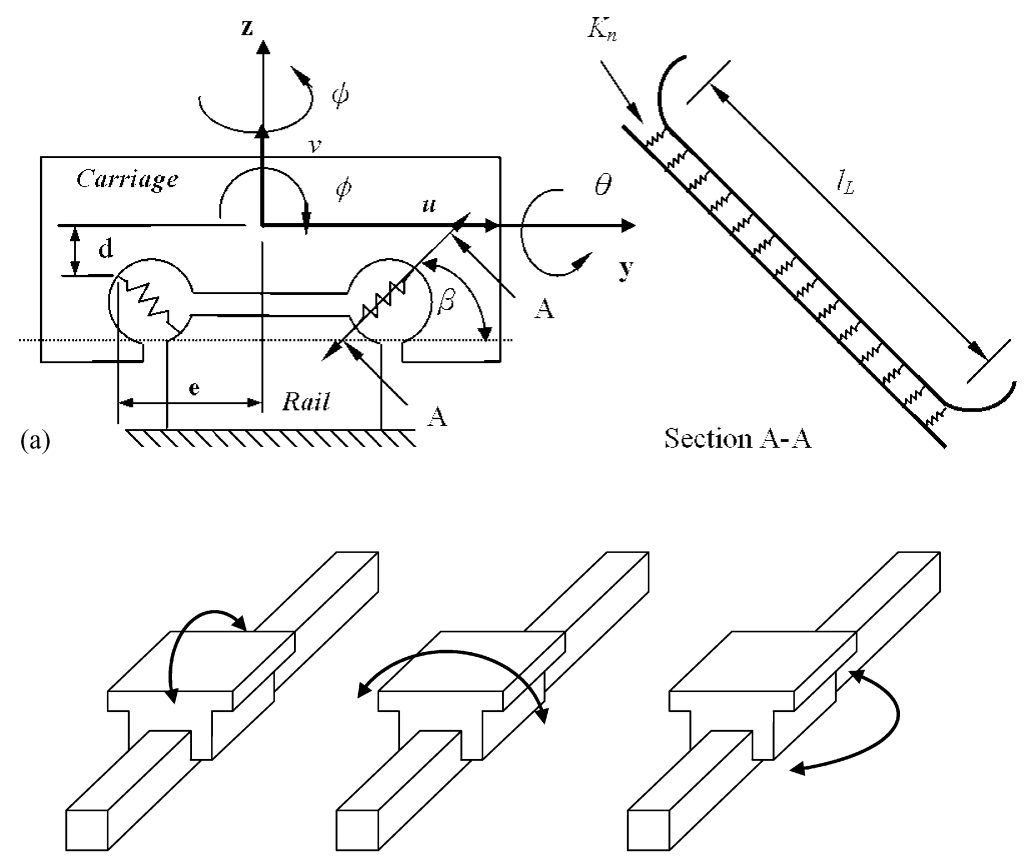

$\begin{array}{lll}\text { (b) }(1) \text { Pitching }(\theta) & \text { (2) Rolling }(\phi) & \text { (3) Yawing }(\psi)\end{array}$

Fig. 2. Coordinate system describing the motion mode of carriage, in which $\beta$ is the contact angle, $u$ and $v$ are the displacements in the $y$ - and $z$-axes, respectively. $\phi, \theta$ and $\psi$ are the angular displacements about the $x$-axis, $y$-axis and $z$-axis, which are termed pitching, rolling and yawing motion, respectively (NSK Ltd.).

coordinate is located at the mass center of carriage block and the sliding direction of carriage block is along the $x$-axis, $\beta$ is the contact angle of rolling ball, $d$ and $e$ are distances of the contact points between rolling ball and carriage to $x-y$ plane and $x-z$ plane, respectively, and $l_{L}$ is the length of the loading zone of balls. For such a spring-mass system, the vibration modes associate with the motion degrees of freedom. In Fig. $2 b$, the motion in vertical direction is termed the vertical vibration mode, the rocking motion $\theta$ about $y$-axis is termed the pitching vibration mode, the rocking motion $\psi$ about $z$-axis is termed the yawing vibration mode and the rocking motion $\phi$ about $x$-axis is termed the rolling vibration mode.

Since the linear spring is perpendicular to the $x$-axis, the displacement along $x$-axis is ignored. In addition, in Fig. 3 , two spring elements are introduced at location of ball bearing and are quantified with the stiffness $K_{\mathrm{n}}$ and $K_{\mathrm{S}}$ in contact normal and tangential direction, respectively, which is the contact stiffness of rolling ball against the raceway groove and is determined based on Hertzian theory. By this simplified model and follow Ohta and Hayashi's deriveations [9], the governing equations of five degrees of freedom model are derived by applying the Lagrange's approach on the potential energy of linear guideway system.

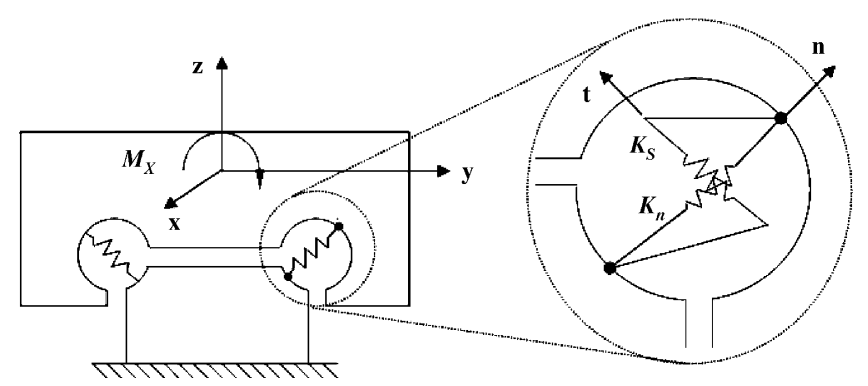

Fig. 3. Modeling of the rolling contact by using a spring element with normal stiffness $K_{\mathrm{n}}$ and another spring element with tangential stiffness $K_{\mathrm{s}}$. 


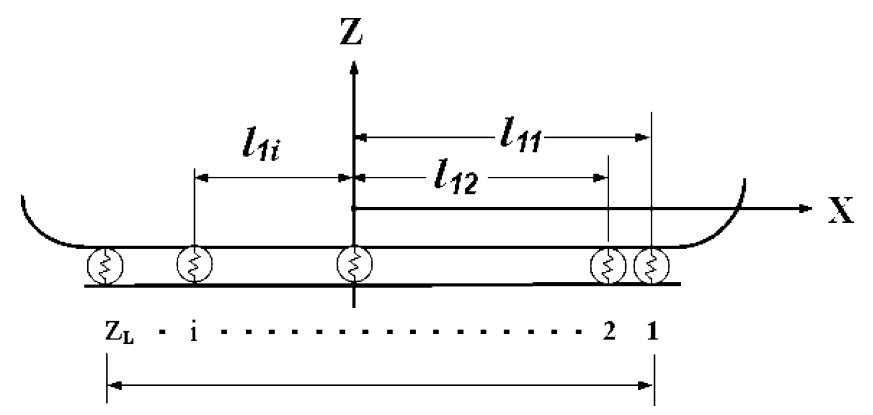

Fig. 4. Location of the $i$ th ball or spring element at right row or left row of raceways, which is measured from $y-z$ plane.

The total kinetic energy $E_{K}$ can be expressed as:

$$
E_{K}=\frac{1}{2} M \dot{u}^{2}+\frac{1}{2} M \dot{v}^{2}+\frac{1}{2} J_{x} \dot{\phi}^{2}+\frac{1}{2} J_{y} \dot{\theta}^{2}+\frac{1}{2} J_{x} \dot{\psi}^{2}
$$

The potential energy $E_{P}$ is given by:

$$
\begin{aligned}
E_{P}= & E_{P 1}+E_{P 2}=\frac{1}{2} K_{n} \sum_{i=1}^{Z_{L}}\left(\delta_{1 i}^{2}+\delta_{2 i}^{2}\right)+\frac{1}{2} K_{s} \sum_{i=1}^{Z_{\mathrm{L}}}\left(\delta_{1 i}^{2}+\delta_{2 i}^{2}\right) \\
E_{P 1}= & \frac{1}{2} K_{\mathrm{n}} \sum_{i=1}^{Z_{L}}\left[\left\{\left(u-d \phi+l_{1 i} \psi\right) \cos \beta+\left(-v-e \phi+l_{1 i} \theta\right) \sin \beta\right\}^{2}\right. \\
& \left.+\left\{\left(-u+d \phi-l_{2 i} \psi\right) \cos \beta+\left(-v+e \phi+l_{2 i} \theta\right) \sin \beta\right\}^{2}\right] \\
E_{P 2}= & \frac{1}{2} K_{s} \sum_{i=1}^{Z_{L}}\left[\left\{\left(u-d \phi+l_{1 i} \psi\right) \sin \beta+\left(v+e \phi-l_{1 i} \theta\right) \cos \beta\right\}^{2}\right. \\
& \left.+\left\{\left(u-d \phi+l_{2 i} \psi\right) \sin \beta+\left(-v+e \phi+l_{2 i} \theta\right) \cos \beta\right\}^{2}\right]
\end{aligned}
$$

where

$$
\begin{aligned}
& Z_{L 1}=Z_{L 2}=Z_{L} \\
& l_{1 i}, l_{2 i}=\left(\frac{Z_{L}-2 i+1}{2 Z_{L}}\right) l_{L}
\end{aligned}
$$

where $E_{P 1}$ and $E_{P 2}$ are the potential energy of normal spring and tangent spring either, $Z_{L 1}$ and $Z_{L 2}$ the average number of balls existing at the left and right load zone, $l_{1 i}, l_{2 i}$ are the location of the $i$ th ball or spring element measured from $y-z$ plane where the subscripts 1 and 2 represent the left and right row of raceways, as shown in Fig. 4.

By applying the Lagrange's approach to Eqs. (8) and (9), we can derive the motion equation corresponding to different degree of freedom as

$$
\begin{aligned}
M \ddot{u}+ & \left\{2 Z_{L} K_{\mathrm{n}} \cos ^{2} \beta+2 Z_{L} K_{\mathrm{S}} \sin ^{2} \beta\right\} u+\left\{-2 Z_{L} K_{\mathrm{n}} d \cos ^{2} \beta\right. \\
- & \left.2 Z_{L} K_{\mathrm{n}} e \sin \beta \cos \beta-2 Z_{L} K_{\mathrm{s}} d \sin ^{2} \beta+2 Z_{L} K_{\mathrm{s}} e \sin \beta \cos \beta\right\} \phi=0 \\
M \ddot{v}+ & \left\{2 Z_{L} K_{\mathrm{n}} \sin ^{2} \beta+2 Z_{L} K_{\mathrm{S}} \cos ^{2} \beta\right\} v=0 \\
J_{x} \ddot{\phi}+ & \left\{-2 Z_{L} K_{\mathrm{n}} d \cos ^{2} \beta-2 Z_{L} K_{\mathrm{n}} e \sin \beta \cos \beta-2 Z_{L} K_{\mathrm{s}} d \sin ^{2} \beta+2 Z_{L} K_{\mathrm{s}} e \sin \beta \cos \beta\right\} u \\
& +\left\{2 Z_{L} K_{\mathrm{n}} d^{2} \cos ^{2} \beta+4 Z_{L} K_{\mathrm{n}} d e \sin \beta \cos \beta+2 Z_{L} K_{\mathrm{n}} e^{2} \sin ^{2} \beta\right\} \phi \\
& +\left\{2 Z_{L} K_{s} d^{2} \sin ^{2} \beta-4 Z_{L} K_{s} d e \sin \beta \cos \beta+2 Z_{L} K_{s} e^{2} \cos ^{2} \beta\right\} \phi=0
\end{aligned}
$$




$$
\begin{aligned}
& J_{y} \ddot{\theta}+\left\{\left(K_{\mathrm{n}} \sin ^{2} \beta+K_{\mathrm{s}} \cos ^{2} \beta\right) \sum_{i=1}^{Z_{L}}\left(l_{1 i}^{2}+l_{2 i}^{2}\right)\right\} \theta=0 \\
& J_{z} \ddot{\psi}+\left\{\left(K_{\mathrm{n}} \cos ^{2} \beta+K_{\mathrm{s}} \sin ^{2} \beta\right) \sum_{i=1}^{Z_{L}}\left(l_{1 i}^{2}+l_{2 i}^{2}\right)\right\} \psi=0
\end{aligned}
$$

In the above, Eq. (15) governs the displacement $v$ in the $z$-axis, Eq. (17) governs the angular displacement $\theta$ about $y$-axis and Eq. (18) describes the angular displacement $\psi$ about $z$-axis. The natural frequencies can then be obtained as calculated from these equations, namely,

The vertical natural frequency of the vibration of the carriage is equal to

$$
f_{\mathrm{V}}=\frac{1}{\pi} \sqrt{\frac{Z_{L} K_{\mathrm{n}} \sin ^{2} \beta+Z_{L} K_{\mathrm{s}} \cos ^{2} \beta}{2 M}}
$$

The pitching natural frequency of the vibration of the carriage equals to

$$
f_{\mathrm{P}}=\frac{1}{2 \pi} \sqrt{\frac{K_{\mathrm{n}} \sin ^{2} \beta+K_{\mathrm{S}} \cos ^{2} \beta}{J_{Y}} \sum_{i=1}^{Z_{L}}\left(l_{1 i}^{2}+l_{2 i}^{2}\right)}
$$

And, the yawing natural frequency of the vibration of the carriage is equal to

$$
f_{Y}=\frac{1}{2 \pi} \sqrt{\frac{K_{\mathrm{n}} \cos ^{2} \beta+K_{\mathrm{s}} \sin ^{2} \beta}{J_{z}} \sum_{i=1}^{Z_{L}}\left(l_{1 i}^{2}+l_{2 i}^{2}\right)}
$$

Since the displacement $u$ along the $y$-axis is coupled with the angular displacement $\phi$ about $x$-axis, we may assume solution is of the form:

$$
\left.\begin{array}{l}
u=U e^{j \omega t} \\
\phi=\Phi e^{j \omega t}
\end{array}\right\}
$$

where $\omega$ is the angular frequency, $j=\sqrt{-1}, U$ and $\Phi$ are the amplitude of $u$ and $\phi$, respectively.

Substituting Eq. (22) into Eqs. (14) and (16), we obtain the followings

$$
\left[\begin{array}{cc}
c_{1}-M \omega^{2} & c_{2} \\
c_{2} & c_{3}-J_{x} \omega^{2}
\end{array}\right]\left[\begin{array}{l}
U \\
\Phi
\end{array}\right]=0
$$

where

$$
\left.\begin{array}{c}
c_{1}=2 Z_{L} K_{\mathrm{n}} \cos ^{2} \beta+2 Z_{L} K_{\mathrm{S}} \sin ^{2} \beta \\
c_{2}=-2 Z_{L} K_{\mathrm{n}} d \cos ^{2} \beta-2 Z_{L} K_{\mathrm{n}} e \sin \beta \cos \beta-2 Z_{L} K_{s} d \sin ^{2} \beta+2 Z_{L} K_{s} e \sin \beta \cos \beta \\
c_{3}=2 Z_{L} K_{\mathrm{n}} d^{2} \cos ^{2} \beta+4 Z_{L} K_{\mathrm{n}} d e \sin \beta \cos \beta+2 Z_{L} K_{n} e^{2} \sin ^{2} \beta \\
+2 Z_{L} K_{\mathrm{S}} d^{2} \sin ^{2} \beta-4 Z_{L} K_{s} d e \sin \beta \cos \beta+2 Z_{L} K_{\mathrm{s}} e^{2} \cos ^{2} \beta
\end{array}\right\}
$$

Make the determinant of the coefficient of $U$ and $\Phi$ equal to zero, we have

$$
M J_{x} \omega^{4}-\left(c_{3} M+c_{1} J_{x}\right) \omega^{2}+c_{1} c_{3}-c_{2}^{2}=0
$$

The two solutions $\omega_{1}^{2}$ and $\omega_{2}^{2}\left(\omega_{1}^{2}<\omega_{2}^{2}\right)$ can be obtained by solving above equation as

$$
\omega_{1,2}^{2}=\frac{c_{3} M+c_{1} J_{x} \mp \sqrt{\left(c_{3} M+c_{1} J_{x}\right)^{2}-4 M J_{x}\left(c_{1} c_{3}-c_{2}^{2}\right)}}{2 M J_{x}}
$$

The other coupled vibration modes of the motion in $u$ and $\phi$ are $f_{\mathrm{RL}}=\omega_{1} / 2 \pi$ and $f_{\mathrm{RH}}=\omega_{2} / 2 \pi$, which are termed the lower rolling and the higher rolling vibration modes of carriage, respectively. In Eqs. (19)-(21) and (26), $M$ is the mass of the carriage, $J_{x}, J_{y}$ and $J_{z}$ are the polar moments of inertia about the $x$-axis, $y$-axis and $z$-axis, respectively. 


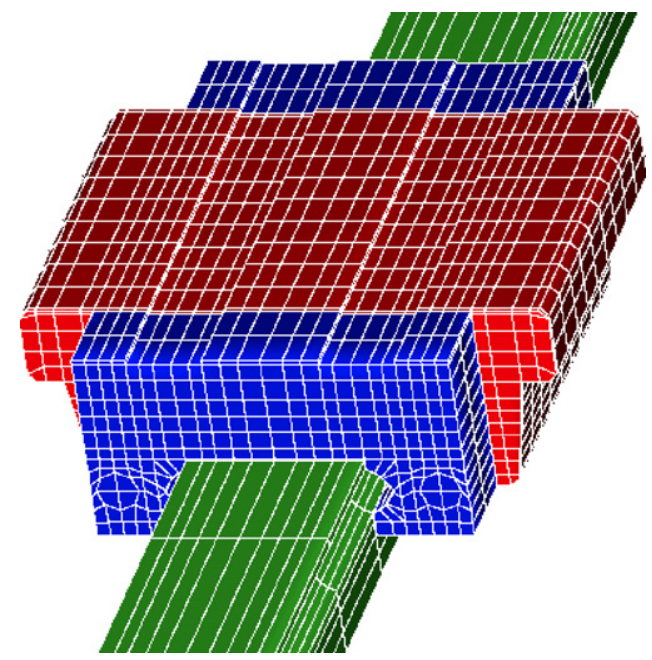

Fig. 5. The finite element model of linear guide system, including rail, carriage, rolling ball and end caps.

It is noticed that the neglect of the term $K_{\mathrm{S}}$, the tangential stiffness, in above formulae yields the natural frequencies similar to the formula presented in Ohta and Hayashi [9]. However, the spring element used in their work was only defined by the normal stiffness, but the tangential stiffness was not considered and the frictional effect was neglected. Meanwhile, the normal stiffness was related to the vertical stiffness measured through the stiffness test of a linear guideway (NSK [8]). The simulation of the contact stiffness by using a single spring element in normal contact direction is herein referred to as $1 \mathrm{D}$ two-point contact mode, different from the 2D contact mode proposed in this study.

\subsection{Finite element approach}

Apart from the analytical approach, factors influencing the dynamics of a linear guide system are considered in this study. All components in the linear guideway are assumed to be elastic and the contact status comes from the elastic deformation of the raceway groove and carriage which is considered as a three-dimensional surface contact module. As stated previously, the surface contact considers both the normal and the frictional effects. So, the normal and the shear stiffnesses are both taken into account in our finite element modal analysis.

Fig. 5 shows the 3D finite element model of a linear guideway mechanism; the specification is listed in Table 1. The contact configuration within this mechanism is depicted in Fig. 6, where two rows of the ball activate between carriage and raceway. Each ball is meshed with adequate number of hexahedron elements. To model the contact characteristic at the rolling interface, the 3D membrane contact elements (zero thickness) are implemented to the upper and the lower sides of each ball. The stiffness $K_{\mathrm{n}}$ and $K_{\mathrm{S}}$ of the contact element are calculated according to the method described previously. The carriage blocks with end cap at both sides are modeled with 3830 elements and 480 elements, respectively, while the rail is modeled using 2158 elements. As a whole, there are 6648 elements and 9396 nodes applied in this system for finite element analysis. Material of components such as ball, carriage and rail are made of steel with the properties: Young's modulus $E=206 \mathrm{GPa}$, Poisson's ratio $\nu=0.30$, density $\rho=7800 \mathrm{~kg} / \mathrm{m}^{3}$, while the end caps have the properties: $E=3.57 \mathrm{GPa}$, Poisson ratio $v=0.30$, density $\rho=1400 \mathrm{~kg} / \mathrm{m}^{3}$. In this model, the stiffness $K_{\mathrm{C}}$ was assigned between the carriage block and ball bearing, and the stiffness $K_{\mathrm{R}}$ between the ball bearing and rail. For simplification in computation, all contact surface are assumed to have the same values, namely, $K_{\mathrm{C}}=K_{\mathrm{R}}=K_{\mathrm{n}}$. Besides, in order to investigate the effect of the preload on the contact stiffness and dynamic behaviors, different preloads (one for high preload of $665 \mathrm{~N}$ and low preload of $190 \mathrm{~N}$ for another) are assumed for the linear guideway model. The normal stiffnesses of the contact interface $\left(K_{\mathrm{n}}\right)$ for low preload and high preload are $14.4 \mathrm{~N} / \mu \mathrm{m}$ and $21.7 \mathrm{~N} / \mu \mathrm{m}$, respectively.

\section{Experimental measurement of vibration}

Now, modal tests were conducted to measure the vibration characteristic of workshop supplied guideways. Fig. 7 shows the experimental configuration. Three accelerometers attached to different position on the carriage 
Table 1

Specifications of the linear guide system

\begin{tabular}{lc}
\hline Carriage length & $65.9 \mathrm{~mm}$ \\
Carriage width & $59 \mathrm{~mm}$ \\
Carriage height & $22 \mathrm{~mm}$ \\
Rail length & $500 \mathrm{~mm}$ \\
Rail width & $20 \mathrm{~mm}$ \\
Rail height & $15.5 \mathrm{~mm}$ \\
Diameter of steel ball & $4.763 \mathrm{~mm}$ \\
Total number of balls & 50 \\
Number of row & 2 \\
Contact angle, $\beta$ & $45^{\circ}$ \\
Preload & $190 \mathrm{~N}($ light preload bearing) \\
& $665 \mathrm{~N}($ high preload bearing) \\
Load zone length, $l_{L}$ & $43 \mathrm{~mm}$ \\
Mass of carriage, $M$ & $0.313 \mathrm{~kg}$ \\
Inertial moment about the $x$-axis, $J_{x}$ & $9.938 \times 10^{-5} \mathrm{~kg} \mathrm{~m}^{2}$ \\
Inertial moment about the $y$-axis, $J_{y}$ & $6.136 \times 10^{-5} \mathrm{~kg} \mathrm{~m}^{2}$ \\
Inertial moment about the $z$-axis, $J_{z}$ & $1.394 \times 10^{-4} \mathrm{~kg} \mathrm{~m}^{2}$ \\
$d$ & $7.37 \mathrm{~mm}$ \\
$e$ & $10.3 \mathrm{~mm}$
\end{tabular}

were implemented; accelerometer A was used to measure the vertical vibration of carriage, accelerometer B was for rolling, pitching vibrations and vertical vibration and accelerometer $\mathrm{C}$ was for yawing and rolling vibrations. For investigating the preload effects and further comparison with numerical predictions, two linear guideways with different preloads (high preload of $665 \mathrm{~N}$ and low preload of $190 \mathrm{~N}$ ) were employed. In experiment, the vibration amplitudes were recorded and stored in a digital spectrum analyzer after hammering the carriage at the measured direction.

The vibration spectra corresponding to the three measuring points $\mathrm{A}, \mathrm{B}$ and $\mathrm{C}$ were depicted in Fig. 8. The main peaks of each measurement point were generalized to obtain frequencies and the associated vibration modes can be identified by comparing to the mode shapes predicted by the finite element approach.
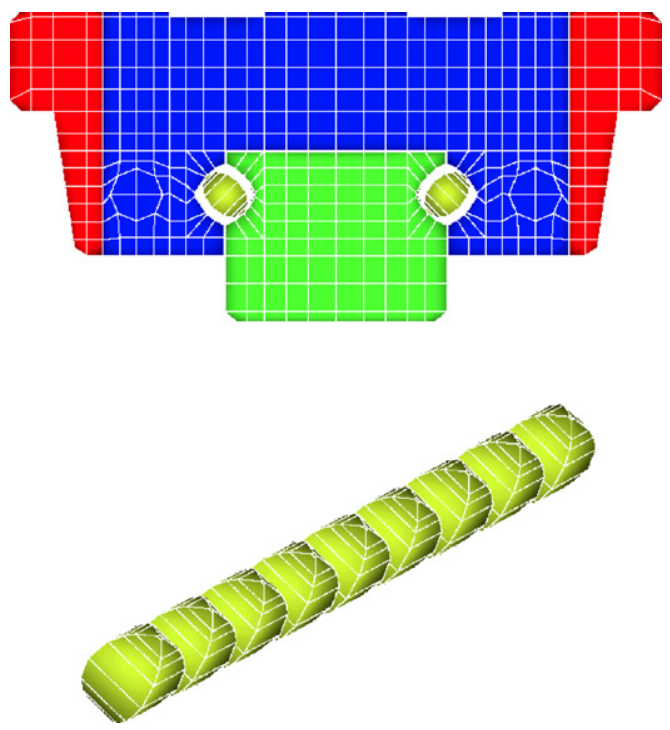

Fig. 6. Finite element mesh of transverse cross section, showing the contact configuration between carriage and rail, in which each rolling ball is meshed using brick elements with a mass equivalent to the ball. 


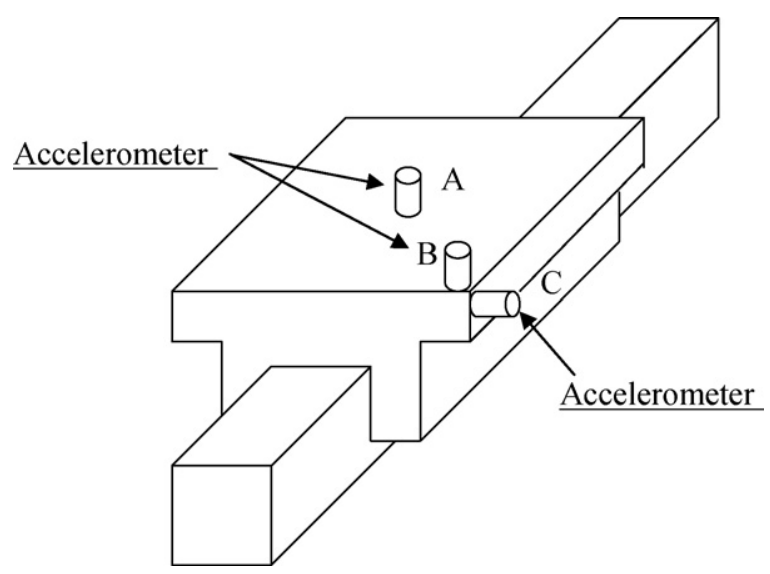

Fig. 7. Configuration of modal experiment and accelerometer positions (A, B and C) for measuring various vibration modes.
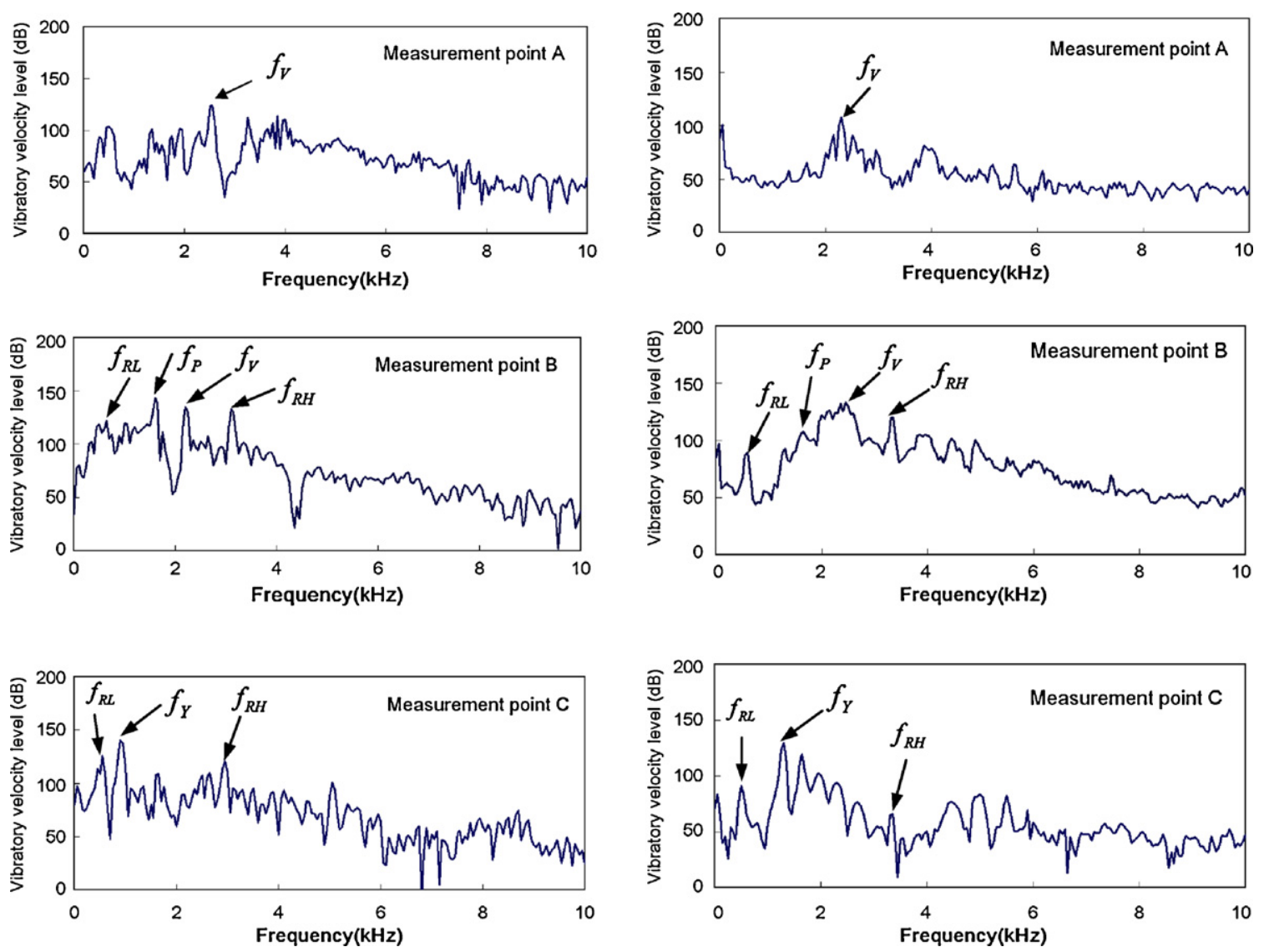

Fig. 8. Vibration spectra of linear guideway with (left) light preload rolling balls and (right) high preload rolling balls.

\section{Results and discussions}

\subsection{Natural frequency of carriage}

The natural frequencies $f_{\mathrm{RL}}, f_{\mathrm{Y}}, f_{\mathrm{p}}, f_{\mathrm{V}}$, and $f_{\mathrm{RH}}$ corresponding to different vibration modes were calculated by substituting the constants in Table 1 into the mathematical models. The results obtained from analytical approach, finite 
Table 2

Natural frequencies of the linear guide with steel balls subjected to (a) light preload and (b) high preload

\begin{tabular}{|c|c|c|c|c|c|c|c|}
\hline \multicolumn{2}{|c|}{ Experimental measurement } & \multicolumn{2}{|c|}{ 1D point-to-point contact model } & \multicolumn{2}{|c|}{ 2D point-to-point contact model } & \multicolumn{2}{|l|}{ FEM } \\
\hline Frequency $(\mathrm{kHz})$ & Vibration mode & $\begin{array}{l}\text { Frequency } \\
(\mathrm{kHz})\end{array}$ & Vibration mode & $\begin{array}{l}\text { Frequency } \\
(\mathrm{kHz})\end{array}$ & Vibration mode & $\begin{array}{l}\text { Frequency } \\
(\mathrm{kHz})\end{array}$ & Vibration mode \\
\hline \multicolumn{8}{|l|}{ (a) Light preload } \\
\hline 0.6 & Lower rolling & - & - & 0.59 & Lower rolling & 0.58 & Lower rolling \\
\hline 1.0 & Yawing & 1.34 & Yawing & 1.41 & Yawing & 1.27 & Yawing \\
\hline 1.8 & Pitching & 2.02 & Pitching & 2.12 & Pitching & 1.81 & Pitching \\
\hline 2.2 & Vertical & 2.29 & Vertical & 2.40 & Vertical & 2.22 & Vertical \\
\hline 3.0 & Higher rolling & 3.23 & Rolling & 3.25 & Higher rolling & 3.02 & Higher rolling \\
\hline \multicolumn{8}{|l|}{ (b) High preload } \\
\hline 0.6 & Lower rolling & - & - & 0.73 & Lower rolling & 0.59 & Lower rolling \\
\hline 1.3 & Yawing & 1.65 & Yawing & 1.73 & Yawing & 1.49 & Yawing \\
\hline 2.1 & Pitching & 2.49 & Pitching & 2.61 & Pitching & 2.14 & Pitching \\
\hline 2.3 & Vertical & 2.81 & Vertical & 2.95 & Vertical & 2.63 & Vertical \\
\hline 3.3 & Higher rolling & 3.97 & Rolling & 4.00 & Higher rolling & 3.64 & Higher rolling \\
\hline
\end{tabular}

element simulation and experimental measurements are summarized in Table 2 and depicted in Fig. 9 for comparison. It is found from Fig. 9 that both the 1D and 2D contact models predict the natural frequencies to be higher than the frequency corresponding to the main peak obtained in modal tests. This can be easily realized because the ball, carriage block and rail were all assumed as rigid bodies and only the deformation of the contact interface were considered in analytical calculation. In addition, the 2D contact model yields an extra vibration mode of lower rolling vibration of the carriage, which is not found in the guideway model with 1D contact model. It seems implying that the contact

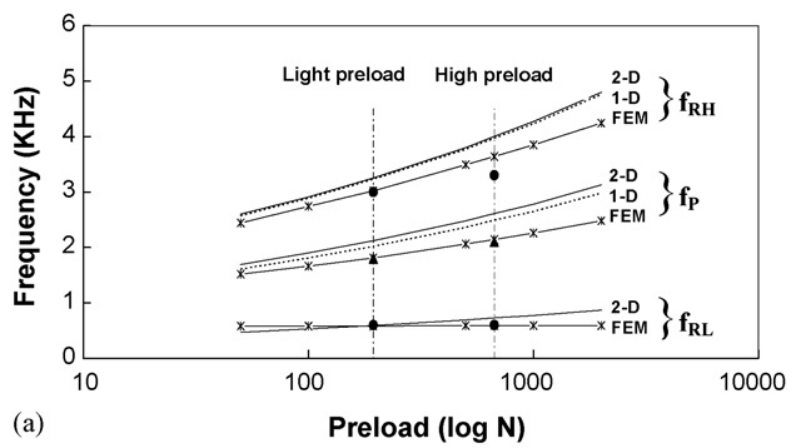

Lower rolling, pitching and higher rolling modes

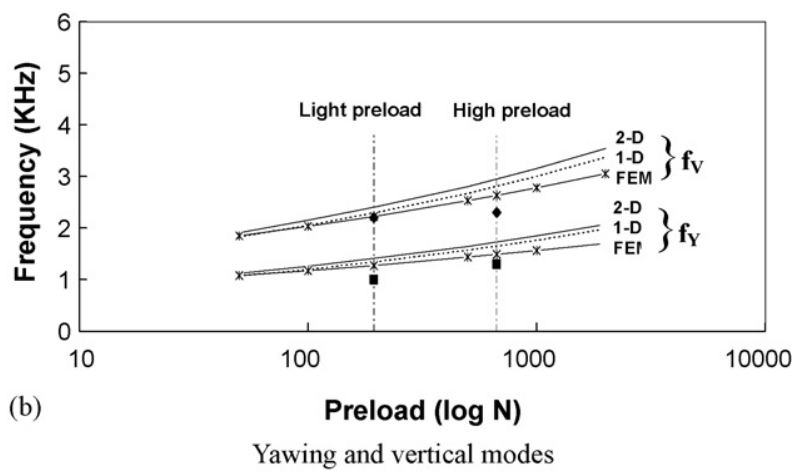

Fig. 9. Comparisons of the natural frequencies of rolling guides with different preloads. Results are obtained from numerical predictions and experimental measurements, respectively, in which the bold symbols $(\mathbf{\bullet}),(\boldsymbol{\Delta}),(\boldsymbol{\square})$, and $(\boldsymbol{})$ represent experimentally measured values. (a) Lower rolling, pitching and higher rolling modes and (b) yawing and vertical modes. 


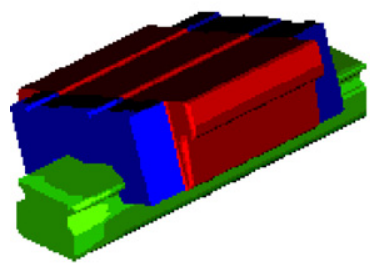

$0.59 \mathrm{kHz}$

( lower rolling mode )

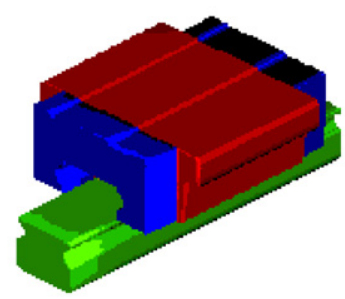

$2.63 \mathrm{kHz}$

( vertical mode )

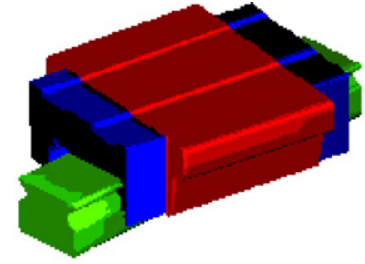

$1.49 \mathrm{kH}$

( yawing mode )

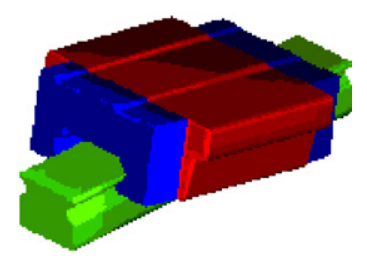

$2.14 \mathrm{kHz}$

(pitching mode )

Fig. 10. Mode shapes of carriage at different vibration frequencies, predicted by finite element analysis.

stiffness in tangential direction due to surface friction affect the dynamic behaviors of linear guideway. Such an effect was also not considered in the 1D model, presented by Ohta and Hayashi [9].

Comparison of results obtained from finite element simulation and analytical calculation also shows that the 2D point-to-point contact model predicts a higher frequency than the finite element method using surface contact model. This is due to the fact that the finite element model was assumed to be an elastic structure and hence possess a lower structural stiffness than a rigid one in analytical approach. Therefore, the finite element approach predicts reasonable results comparable to the experimentally measured data. It is obvious that the surface-to-surface contact mode with Hertzian contact stiffness demonstrates the contact characteristic of the rolling contact interface in realistic way. The prediction of the vibration modes of the linear guide system by the finite element method are further depicted in Fig. 10. In the figure, it clearly shows the variety of mode shapes of a linear guide system, which including the mode shape of yawing, one pitching, one vertical and two rolling vibration and the frequencies of modes are in the sequence from low to high: lower rolling $<$ yawing $<$ pitching $<$ vertical $<$ higher rolling.

\subsection{Effect of preload}

Preload of the rolling elements is generally used to improve the rigidity and accuracy of a guideway (NSK [8] Ltd.). However, the preload produces initial contact force on rolling elements in assemblage. To investigate the effect of preloaded ball on the vibration characteristic, different preloads set on linear guideway were assumed for this analysis here. As shown in Fig. 9, both of the numerical prediction and experimental measurement indicate that the guideway system vibrates at higher frequency when subjected higher preload; while it vibrates at lower frequency under lighter preloading condition. The variation in vibration mode can be ascribed to the change of the contact stiffness at contact interface, which will increase with the increasing preload applied on the carriage of guideway. The effect of the preload on the vibration characteristic of the bearing is thus apparent.

\subsection{Effect of ball material}

To prevent the positioning errors due to surface wear of a rolling element, a ceramic ball with better wear-resistance property was employed to replace the steel ball in linear guideway in this study. As revealed in Hertzian contact theory, 

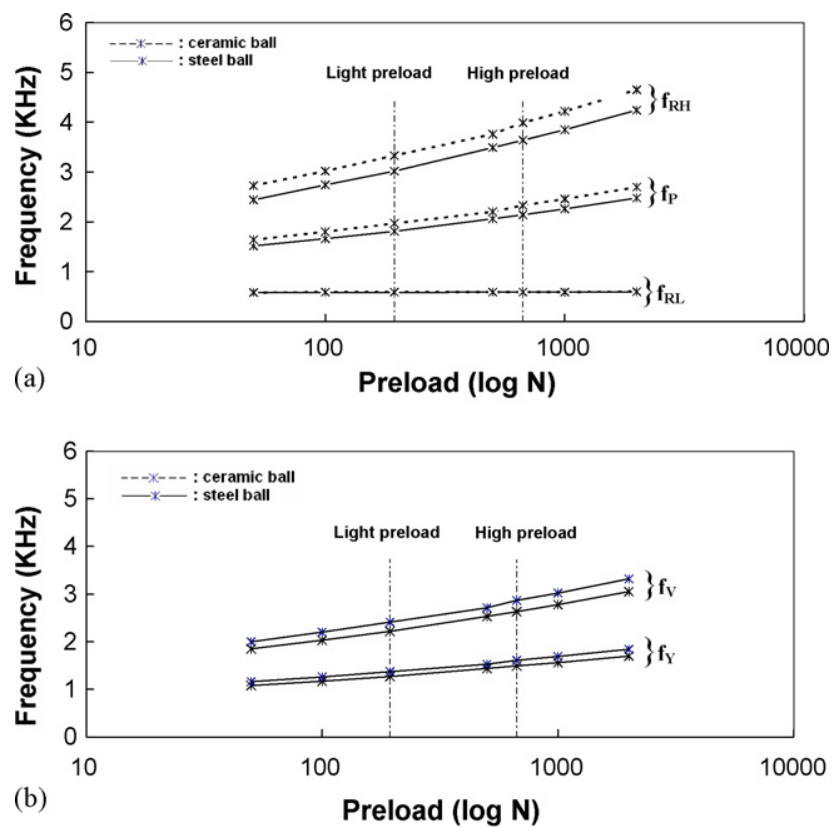

Fig. 11. Comparisons of the natural frequencies of rolling guides with different preloads, results obtained from finite element simulation for guideways with steel ball and ceramic ball, respectively. (a) Lower rolling, pitching and higher rolling modes and (b) yawing and vertical vibration modes.

the material stiffness plays an important role on contact stiffness at the rolling interface. Therefore, the use of ceramic ball was used to expect the improving effect of the contact stiffness at the rolling interface and hence the dynamic behaviors of the structure. In this analysis, the following material properties were used for a ceramic ball: Young's modulus $E=380 \mathrm{GPa}$, Poisson's ratio $\nu=0.30$, density $\rho=3900 \mathrm{~kg} / \mathrm{m}^{3}$. The normal stiffnesses of the contact interface $\left(K_{\mathrm{n}}\right)$ for the low preload and the high preload are $17.1 \mathrm{~N} / \mu \mathrm{m}$ and $25.8 \mathrm{~N} / \mu \mathrm{m}$, either. Because of the change of contact stiffness, the dynamic characteristic of the linear slide rail system could be affected. Fig. 11 and Table 3 show that the natural frequencies obtained for the ceramic ball. It is again found that the nature frequencies obtained from finite element approach are lower than those obtained from analytical approach. Moreover, we compared the variation of nature frequencies of guideway with steel ball and with ceramic ball under the same preload condition and found that

Table 3

Natural frequencies of the linear guide with ceramic balls subjected to (a) light preload and (b) high preload

\begin{tabular}{|c|c|c|c|c|c|}
\hline \multicolumn{2}{|c|}{ 1D point-to-point contact model } & \multicolumn{2}{|c|}{$2 \mathrm{D}$ point-to-point contact model } & \multicolumn{2}{|l|}{ FEM } \\
\hline Frequency $(\mathrm{kHz})$ & Vibration mode & Frequency $(\mathrm{kHz})$ & Vibration mode & Frequency $(\mathrm{kHz})$ & Vibration mode \\
\hline \multicolumn{6}{|l|}{ (a) Light preload } \\
\hline- & - & 0.64 & Lower rolling & 0.59 & Lower rolling \\
\hline 1.46 & Yawing & 1.53 & Yawing & 1.37 & Yawing \\
\hline 2.20 & Pitching & 2.30 & Pitching & 1.96 & Pitching \\
\hline 2.49 & Vertical & 2.61 & Vertical & 2.41 & Vertical \\
\hline 3.50 & Rolling & 3.54 & Higher rolling & 3.31 & Higher rolling \\
\hline \multicolumn{6}{|l|}{ (b) High preload } \\
\hline- & - & 0.79 & Lower rolling & 0.59 & Lower rolling \\
\hline 1.79 & Yawing & 1.88 & Yawing & 1.61 & Yawing \\
\hline 2.70 & Pitching & 2.84 & Pitching & 2.33 & Pitching \\
\hline 3.06 & Vertical & 3.21 & Vertical & 2.86 & Vertical \\
\hline 4.32 & Rolling & 4.35 & Higher rolling & 3.99 & Higher rolling \\
\hline
\end{tabular}


the contact stiffness is increased due to the use of ceramic ball and this in turn shifts the nature frequencies of guideway system to higher values. The effect of material stiffness of a rolling ball on the dynamic characteristics can also be demonstrated from the above results. For a carriage with sufficiently higher Young's modulus, it might behave like a rigid body and hence result in a vibration behavior with higher frequency. Such a status had also been shown in the analytical predictions.

On the other hand, the guideway system discussed in this study is designed with the offset Gothic arc groove, which enables the rolling ball to contact with carriage and raceway simultaneously, and hence only two rolling interfaces can be formed; one is between the rolling ball and the groove of carriage and another is between the rolling ball and the groove of rail. The interface elements with normal and tangential stiffness are thus introduced at upper and lower interfaces of the rolling ball in the finite element model. In practice, a heavy-duty guideway has been designed in workshop with symmetric Gothic arc grooves to sustain a great deal of axial and radial force, which gives a four-point contact configuration at various contact angles. Under such condition, the understanding of contact characteristic at the rolling interface can be obtained by introducing the interface element at the four contact points where the contact stiffness at each interface can be calculated according to the contact configuration formed within the guideway. Besides, the actual contact angle may deviate from the initial contact angle due to the preload applied on the guideway and hence alters the contact stiffness. Current results indeedly demonstrate this status that the preload may bring the contact stiffness of the rolling interface to a different value and affect the dynamic characteristic of a linear guideway. It is therefore necessary to calculate the optimum preload in order to obtain adequate natural frequency of a guideway during operating.

\section{Conclusions}

The contact effect of structures by the three-dimensional finite elements associated with proper theory and modeling techniques was studied here. Modal tests were also experimentally done to understand the significance of the simulation of the dynamic behaviors of linear guideway systems. In the finite element approach, spatial membrane elements (also known as the non-thickness interface) were implemented to simulate the contact interface of the guideway systems and the contact stiffness between steel bearing and carriage/rail, both in the normal and tangential directions were considered with the Hertzian contact theory. The finite element approach provided very good consistencies in the prediction of the dynamic behaviors of the linear guideway systems comparing with the experimental results. In a series of computations, it was found that the contact stiffness used at the interfaces might affect the natural frequency of the linear guide system seriously and such stiffness was relevant to the preload on steel ball, deformation of the interface and the material of the contact objects obviously. Moreover, bearings with higher preloads might present higher frequencies than those with lower preload ones. The natural frequency was also obviously varied with the external loads applied on the guideway systems in which the weight of the carriage block and the assembly preload were kept unchanged. The algorithm studied here can be applied to other structural systems in the process of the design of machine tools easily and the application of different loadings, such as bending moments, distributive load or loads from different directions would be studied in other issues.

\section{References}

[1] J. Egert, Comparison of some contact elements and iterative algorithms for the solution of frictionless contact problems, J. Comput. Appl. Mech. 1 (1) (2000) 23-36.

[2] W. Goldsmith, Impact-the Theory and Physical Behavior of Colliding Solids, Edward Arnold Ltd., 1960.

[3] G.D. Hagiu, M.D. Gafitanu, Dynamic characteristics of high speed angular contact ball bearings, Wear 211 (1997) 22-29.

[4] X. Hernot, M. Sartor, J. Guillot, Calculation of the stiffness matrix of angular contact ball bearings by using the analytical approach, J. Mech. Design 122 (2000) 83-90.

[5] D.H. Hwang, K.H. Gahr, Transition from static to kinetic friction of unlubricated or oil lubricated steel/steel, steel/ceramic and ceramic/ceramic pairs, Wear 255 (2003) 365-375.

[6] K.J. Johnson, Contact Mechanics, Cambridge University Press, 1985.

[7] N. Lynagh, H. Rahnejat, M. Erahimi, R. Aini, Bearing induced vibration in precision high speed routing spindle, Int. J. Mach. Tools Manuf. 40 (2000) 561-577.

[8] NSK Ltd., Selection Guide to NSK Linear Guides.

[9] H. Ohta, E. Hayashi, Vibration of linear guideway type recirculating linear ball bearings, J. Sound Vib. 235 (5) (2000) $847-861$. 
[10] H. Ohta, Sound of guideway type recirculating linear ball bearings, Trans. ASME J. Tribol. 121 (1999) 678-685.

[11] J. Perret-Liaudet, Subharmonic resonance of order 2 on a sphere-plan contact, C. R. Acad. Sci. Paris Series II b325 (1997) $443-448$.

[12] J. Perret-Liaudet, Superharmonic resonance of order two on a sphere-plan contact, C. R. Acad. Sci. Paris Series II b326 (1998) 787-792.

[13] M. Pimsarn, K. Kazerounian, Efficient evaluation of spur gear tooth mesh load using pseudo-interference stiffness estimation method, Mechan. Mach. Theory 37 (2002) 769-786.

[14] J.H. Yeh, F.W. Liou, Contact condition modelling for machining fixture setup processes, Int. J. Mach. Tools Manuf. 39 (1999) $787-803$. 